\title{
A Skin Fixation Method for Decreasing the Influence of Wound Contraction on Wound Healing in a Rat Model
}

\author{
Seong Hwan Bae, Yong Chan Bae, Su Bong Nam, Soo Jong Choi \\ Department of Plastic and Reconstructive Surgery, Pusan National University School of Medicine, Busan, Korea
}

\begin{abstract}
Background The elasticity of the back skin of the rat reduced the tension around wounds during the wound healing process in that region, and thus activates wound contraction. The authors proposed two skin fixation methods using readily available materials to decrease the influence of wound contraction on wound healing and designed an experiment to determine their effects.

Methods The authors made 36 skin wounds on the backs of 18 rats, and they divided them into three groups. Each group was treated with three different kinds of dressing materials, each with different skin fixing characteristics. Group A was a control group. Group B and group $C$ were dressed by the first and the second skin fixation method. We measured the areas of the wounds post-surgically and calculated the wound area reduction rates.

Results The two skin fixation methods both reduced the effect of wound contraction compared to the control group. Each of the two methods had different outcomes in reducing wound contraction.

Conclusions The experiment demonstrated significant differences among the wound areas and the wound area reduction rates of the three groups as a result of differences in the degree of wound contraction. To obtain accurate results from wound healing experiments, appropriate skin fixation methods must be adopted.
\end{abstract}

Keywords Wounds and injuries / Wound healing / Rats
Correspondence: Soo Jong Choi Department of Plastic and Reconstructive Surgery, Pusan National University School of Medicine, 179 Gudeok-ro, Seo-gu, Busan 602-739, Korea Tel: +82-51-240-7826 Fax: +82-51-243-9405 E-mail: drsjong@gmail.com

\section{INTRODUCTION}

In general, the wound healing process can be classified into three different phases: the inflammatory phase, proliferative phase, and remodeling phase. This process is a series of complicated reactions and interactions among cells and mediators, which can be affected by various factors [1]. Among these, the proliferative phase is characterized by fibroplasia, granulation, contraction, and epithelialization. Contraction is the process in which the surrounding skin is pulled circumferentially toward an open wound, which reduces the wound without forming new tissues, speeds up the wound healing, and minimizes scar formation.

Rats are one of the experimental animals that are widely used for wound healing experiments, so a number of such studies have been published. Most rat wound healing experiments use the back skin of the rats since the panniculus carnosus located under the subcutaneous fat is loosely combined with the deeper muscle layer. Such characteristics of the back skin of rats reduce

Copyright () 2012 The Korean Society of Plastic and Reconstructive Surgeons

This is an Open Access article distributed under the terms of the Creative Commons Attribution Non-Commercial License (http://creativecommons.org/

licenses/by-nc/3.0/) which permits unrestricted non-commercial use, distribution, and reproduction in any medium, provided the original work is properly cited.

www.e-aps.org 
the tension around wounds in the wound healing process, and thus activate wound contraction [2]. Since minimizing the influence of wound contraction by fixing the skin is essential to obtain accurate experimental results, various skin fixation methods have been designed, based on which a variety of experiments have been conducted [3-6]. Most of these experiments, however, have not compared skin fixation methods, and adopted experimental materials that are not widely available, which made it difficult for other researchers to apply the methods in their experiments. Therefore, the authors present two skin fixation methods that can reduce the effects of wound contraction by means of readily available materials, and that, in comparison with the control group, reduce the influence of wound contraction.

\section{METHODS}

\section{Experimental materials}

\section{Experimental animals}

For the experimental animals, 8-week-old, male, white SpragueDawley rats of 250 to $300 \mathrm{~g}$ on average were used (18 rats). The experiment began after an adjustment period of more than a week in which they were fed with semi-synthetic feed in the lab.

\section{Skin fixation materials}

For this experiment, Tegaderm (3M, St. Paul, MN, USA), Duoderm CGF (ConvaTec, a division of ER Squibb \& Sons, Princeton, NJ, USA), and adhesive polyvinyl chloride film were used. Since Tegaderm is very thin, it might protect the wound from the surroundings, but it could not fix the skin due to its elasticity. Duoderm CGF is thick and adhesive enough to fix the skin to some extent, but the adhesion might weaken over time or the adhered section could be softened or deformed due to exudate, humidity, temperature, and so forth. Adhesive polyvinyl chloride film is made of polyvinyl chloride, and is colorless, transparent, and $0.15 \mathrm{~mm}$ thick, which is likely to bend but hardly wrinkle. This is a laminating paper available in office supply stores. Since it is not too adhesive to hold the skin tightly, the shape is not easily deformed due to surrounding conditions and it has good durability.

\section{Skin fixation methods}

The authors suggest the following two skin fixation methods by means of the skin fixation materials that are relatively common and readily available. The first method is as follows: Duoderm CGF, which has the ability to fix the skin, is attached to the area surrounding the wound, and the Duoderm CGF is covered with a second layer, of Tegaderm. Then Tegaderm, Duoderm CGF, and the back skin of the rats are sutured by means of stitching fibers. This way, the wound is protected from the outside and the skin is fixed. In particular, the fixation by using stitching fibers was expected to prevent the Duoderm CGF from falling off or becoming somewhat deformed. The second method is as follows: as in the first method, the Duoderm CGF is attached to the skin around the wound, and then it is covered by adhesive polyvinyl chloride film instead of Tegaderm, the latter of which has no ability to fix the skin. Then the adhesive polyvinyl chloride film, Duoderm CGF, and the back skin of the rats are sutured by means of stitching fibers. Since this method is made of stronger materials, it causes less deformation than in the first method, and better skin fixation performance was expected.

\section{Experimental methods}

\section{Wound induction and treatment}

Twenty mg/kg of $5 \%$ ketamine hydrochloride (Ketamine HCL, Huons, Seoul, Korea) and $2 \mathrm{mg} / \mathrm{kg}$ of xylazine hydrochloride (Rompun, Bayer Korea, Seoul, Korea) were mixed and injected into the abdominal cavity to put the rats under anesthesia and then the fur on the back skin was removed. The back was then disinfected with $10 \%$ povidone-iodine and $70 \%$ ethanol. One square-shaped wound $(10 \times 10 \mathrm{~mm})$ was made in each side of the back, and the full-thickness skin defect was made up to the top of panniculus carnosus by means of a number 15 scalpel and Metzenbaum tissue scissors (Fig. 1). Thirty-six wounds were cut into 18 white rats, and two wounds from one rat were assigned to two different groups. As a result, three different groups-A, B, and $\mathrm{C}$-were formed, each containing 12 wounds. Among these, group A was designated as the control group and covered with Tegaderm alone, which could protect wounds but had little ability to fix the skin. To group B, the first skin fixation method was applied. With the central part cut out in a square shape $(15 \times 15$ $\mathrm{mm}$ ), Duoderm CGF was put around the wound spanning the same area, covered with Tegaderm, and then fixed at 8 points

\section{Fig. 1. Photographs of a rat}

After the dorsal hair was removed, two full-thickness skin wounds were made on the back of each rat.

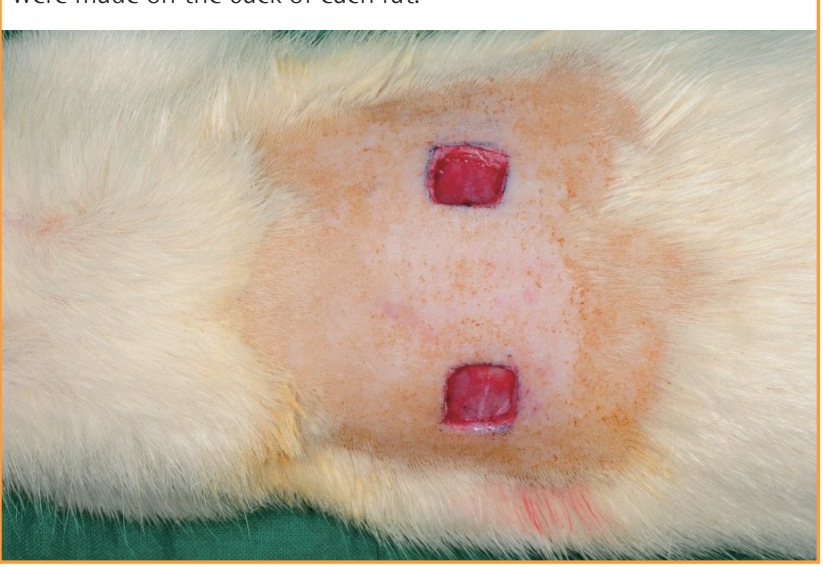


with nylon 7-0. To group $C$, the second skin fixation method was applied. As in group B, Duoderm CGF was attached, but this time it was covered with an adhesive polyvinyl chloride film, and then fixed with nylon 7-0 (Fig. 2).

For all rats, the dressed wounds were wrapped three times in Peha-Haft (Paul Hartmann AG, Heidenheim, Germany) regardless of the skin fixation method, and then wrapped again twice with Ioban (3M). This way, any possible damage to the experimental material was prevented, and excessive movement of the rats was also controlled to restrict wound contraction and thus minimize errors. One breeding cage was designated for each rat so as to prevent the dressing from being damaged by other rats.

\section{Wound area and wound area reduction rate measurements}

On the 3rd, 6th, 9th, and 12th days of the experiment, the size of the skin defect on the wound areas was photographed by means of a digital camera with the same camera settings and under the same conditions each time. Then the borders of the skin defect in the pictures were marked, and then the number of pixels within the bordered area was measured to calculate the wound area by using Photoshop CS5 software (Adobe, San Jose, CA, USA). Based on the measured wound area, the wound area reduction rate was calculated by means of the following expression.

former size of the wound-current size of the wound former size of the wound

\section{Statistical analysis}

Based on the results of each group, the wound areas of each group on each date and the statistical differences in the wound area reduction rates were analyzed by one-way ANOVA and multiple comparison using SPSS ver. 14.0 (SPSS Inc., Chicago,
IL, USA). In a multiple comparison, the group that satisfied the requirement in terms of equal variance was analyzed by means of Tukey's method while the others were analyzed using the Dunnett T3 method. When P was less than 0.05 , it was regarded as statistically significant.

\section{RESULTS}

\section{Wound area}

The average wound area and standard deviation on each date for each group in this experiment are presented in Table 1. Three

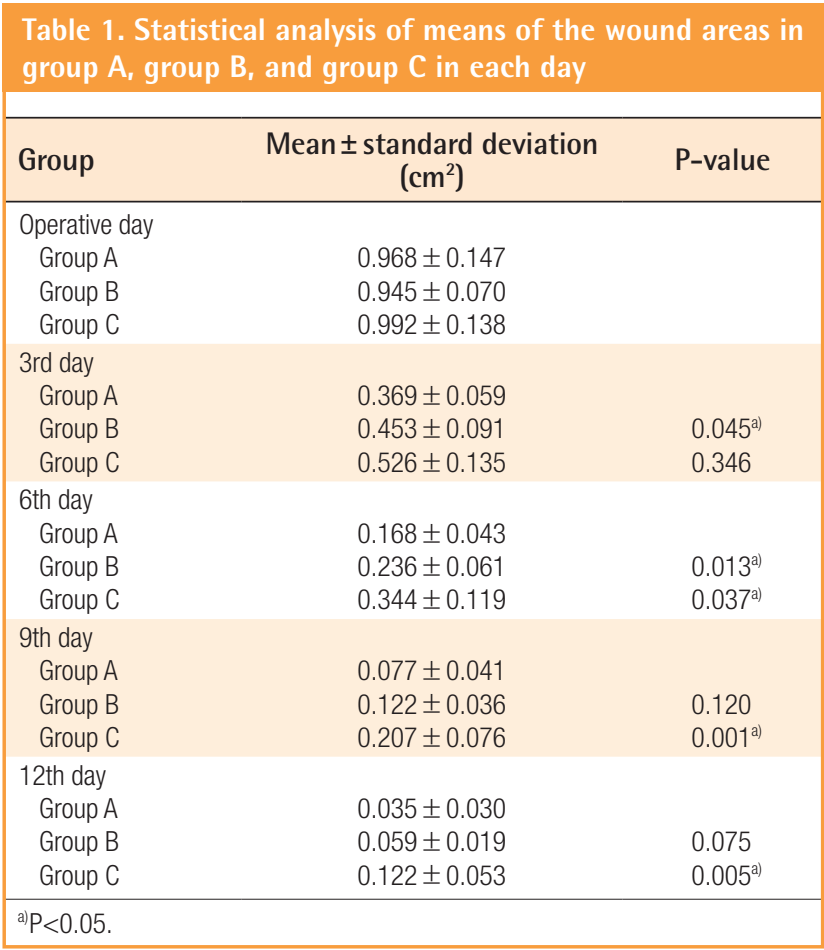

\section{Fig. 2. Three different kinds of skin fixation methods}

Photographs of the wounds that were each dressed by one of three different skin fixation methods. (A) Group A was dressed by Tegaderm alone. (B) Group B was dressed by Duoderm CGF, Tegaderm, and nylon 7-0. (C) Group C was dressed by Duoderm CGF, adhesive polyvinyl chloride film, and nylon 7-0.
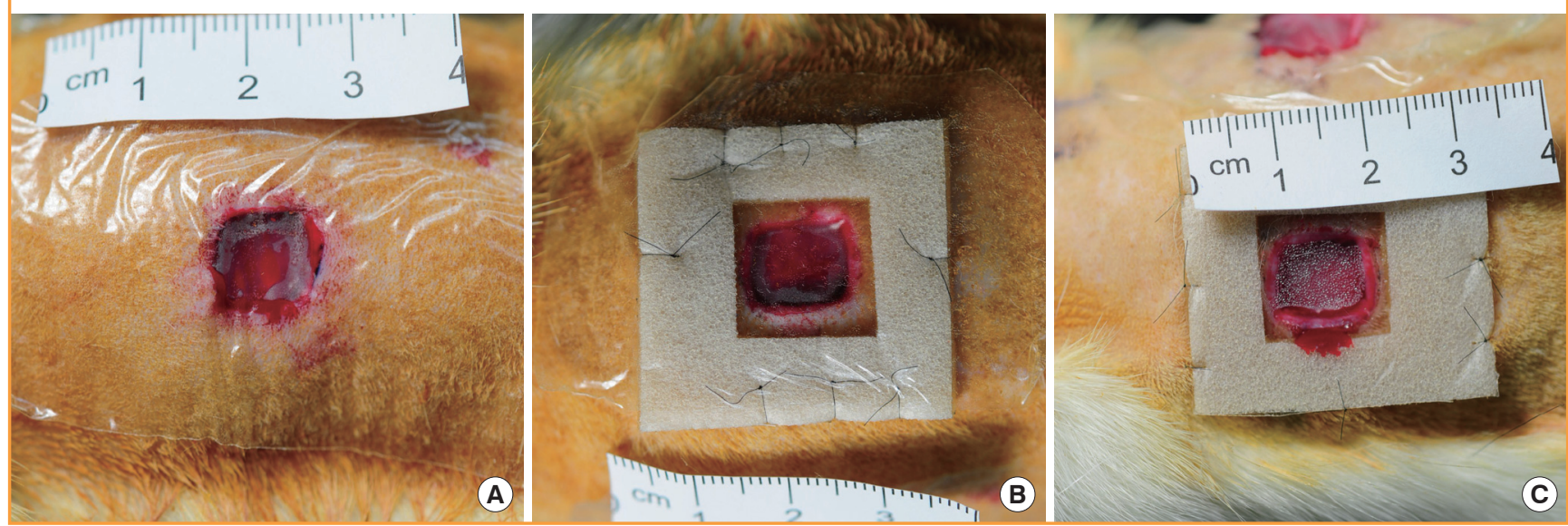


\section{Fig. 3. Wound area reduction rates in each periods}

Averages and standard deviations of the wound area reduction rates by the three different skin fixation methods in each period. After the wound was made, for days $0-3$, days $3-6$, and days $6-9$, group $A$ had the higher wound area reduction rate than group $C(P<0.05)$ in all cases, and there was no difference between groups $A$ and $B$, or between groups $B$ and $C(P>0.05)$. On days $9-12$, there was no difference among groups $A, B$, and $C(P>0.05)$.

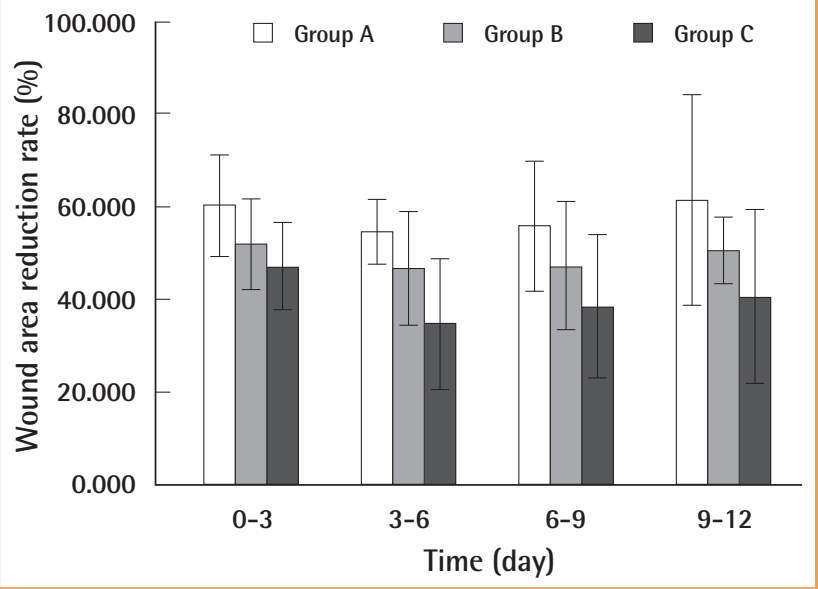

days after the wound was made, the area of group A was smaller than those of groups $B$ and $C(P<0.05)$, while there was little difference between group $\mathrm{B}$ and group $\mathrm{C}(\mathrm{P}=0.346)$. On the 6th day, the wound area of group A was smaller than that of group $B(P=0.013)$, while that of group $B$ was smaller than that of group $\mathrm{C}(\mathrm{P}=0.037)$. In short, the size of wounds was in the order of group $\mathrm{A}$, group $\mathrm{B}$, and group $\mathrm{C}$ from smallest to largest. On the 9th day, the wound of group $C$ was bigger than those of group $\mathrm{A}$ and group $\mathrm{B}(\mathrm{P}<0.05)$, while there was no significant difference between group $A$ and group $B(P=0.120)$. On the 12th day, as on the 9th day, the wound area of group $C$ was bigger than those of group $\mathrm{A}$ and group $\mathrm{B}(\mathrm{P}<0.05)$, while there was no difference between group $A$ and group $B(P=0.075)$.

\section{The wound area reduction rate}

The average and the standard deviation of the wound area reduction rate in each period and in each group are presented in Fig. 3. After the wound was made, for days 0-3, days 3-6, and days 6-9, group A had a higher wound area reduction rate than group $\mathrm{C}(\mathrm{P}<0.05)$ in all cases, and there was no difference between groups $A$ and $B$, or between groups $B$ and $C(P>0.05)$. On days 9-12, there was no difference among groups $A, B$, and $\mathrm{C}(\mathrm{P}>0.05)$.

\section{DISCUSSION}

In wound healing, a wound closure takes place through the formation of a scar as a result of accumulation of substrates that have properties similar to those of the dermis at the damaged skin, which contributes to recovering the continuity of the skin. In this process, wound contraction by the surrounding dermis and fat tissues plays an important role [7]. It is known that wound contraction results from the pulling of dermis and fat tissues as collagen fibrils are concentrated with cells such as fibroblasts, become collagen fibers, and again become granuloma tissue, which reduces the volume $[7,8]$. Unlike the human wound healing process, experimental animals such as rats experience less tension of the skin pulling around the wound due to looser skin. Thus, active wound contraction, as in this experiment, plays an important role in healing [9]. In this experiment, it turned out that the progress of wound healing was slow in the groups in which adhesive polyvinyl chloride film or Duoderm CGF was used to fix the loose skin and to stabilize the skin tension.

It has been demonstrated in a number of studies that as above, the fixation of skin around a wound could affect the wound healing rate because of the decreased influence of contraction in the process of wound healing. Green et al. [10] and Ehrlich and Needle [2] reported difference in the duration of wound healing in mice with a tight-skin mutation compared with normal rats.

Engin et al. [11] found that removing nerves around the wound reduces the wound contraction and thus slows down the wound healing. In a rat wound healing experiment, therefore, fixing the skin around the wound could be of great importance, and thus various skin fixation methods have been designed to this effect. Korula et al. [3], for example, attempted to prevent wound contraction by means of adherent splints, while Callaghan et al. [4] and Lin et al. [5] sutured wounds with nylon on a silicone splint. Lindenblatt et al. [6] used a titanium splint to reduce errors due to wound contraction. However, most such methods did not show a reduction in wound contraction relative to a control group. Further, these methods were designed with materials that are not readily available to other researchers. Thus, the authors designed a new method for reducing the effect of wound contraction simply and economically, and conducted an experiment to compare this method with a control group to demonstrate that of the method could reduce wound contraction.

In this experiment, the authors attached Duoderm CGF $(15 \times 15 \mathrm{~mm})$ over the $10 \times 10 \mathrm{~mm}$ wound in group $B$ in order to stabilize the tension of the area around the wounded skin. In group C, $15 \times 15 \mathrm{~mm}$ Duoderm CGF was also attached to the wound, but then an adhesive polyvinyl chloride film was applied over it in order to provide stronger force than that applied to group $B$ to further reduce any error arising from wound contraction. The $2.5 \mathrm{~mm}$ wide gap between the borders of the wound and Duoderm CGF served to fix the skin around the wound and minimize the effect of the Duoderm CGF at the same time. Fur- 
ther, Peha-Haft and Ioban were also used to prevent the dressing materials from being damaged or lost. Also, when the authors took photographs or handled the rats, inhalation anesthesia by means of isoflurane was used. Furthermore, the initially attached dressing material was kept unchanged in order to minimize the effect of unexpected factors that might take place while handling the rats, and also to maintain the humidity $[12,13]$. Thus the number of variables that could affect the results of the experiment was reduced as much as possible and wound healing could proceed undeterred.

Among the dressing materials used in this experiment, Tegaderm is made of polyurethane, which protects the wound from external conditions and prevents microorganisms from penetrating. In addition, its adhesive makes it easy to apply to the skin. Duoderm CGF is a dressing material that contains hydrocolloid. It is known that this material can absorb wound exudate; maintain the humidity, which helps the wounded skin recover; and remove foreign substances and necrotic tissue. In addition, as Duoderm CGF adheres well to the skin around a wound, the wounded area could be completely isolated from the external environment, and no dead space exists between the wound and dressing, which limits the reproduction of microorganisms. Thus, in this experiment, the authors used this material because it is a more rigid material with adherence than Tegaderm.

As for the adhesive polyvinyl chloride film, laminating films that are available in regular office supply stores were used. Although this film is not a dressing material, it was used in this experiment to fix the skin with sufficient force. Polyvinyl chloride, which is a component of adhesive polyvinyl chloride film, softens at $65^{\circ} \mathrm{C}$ or higher and is stable in the solid state at room temperature, and the flexibility may change depending on the plasticizer. This material is utilized for various products such as film, toys, food containers, and so forth. In this experiment, the authors used this material because it is the most rigid among these three kinds of materials.

In this experiment, it can be said that the skin-fixing strength is in the order of group A, group B, and group C. As a result of the experiment, it turned out that the skin-fixing force affects the area of the wounded skin and wound area reduction rate, which indicates that preventing wound contraction could result in a significant decrease in the wound healing speed. Meanwhile, between $B$ and $C$, a difference in the area of the wounded skin was observed, which shows that the group $\mathrm{C}$ approach better prevented wound contraction in the wound healing process than that of group B. Hence, it would be most desirable to fix the skin with a material that is durable enough to prevent deformation during the period of experimentation, as in the case of group C. This experiment shows that the results of rat wound healing ex- periemnts might not be reliable unless the back skin of the rats is properly fixed during wound healing using an adequate skin fixation method to prevent wound contraction from influencing wound size.

However, the effect of the dressing materials, that is, the Tegaderm, Duoderm CGF, and adhesive polyvinyl chloride film, on the wound was not taken into consideration. Nor was the effect of the pressure by the Peha-Haft and Ioban considered in this study, which could be important variables as well. It is thought, therefore, that additional studies on these factors should be performed. In addition, the method that the authors designed basically fixes the dressing materials on the back skin of rats by using suturing fibers, which cannot be applied to experiments where the wound should be regularly opened and sterilized.

For the wound healing experiment, the authors used materials that are commonly available in one of two proposed methods of fixing the skin around the wound in order to reduce the effect of wound contraction. The wound healing experiment was conducted by means of experimental rats, and it turned out that the two skin fixation methods reduced the effect of wound contraction compared to the control group in which only Tegaderm was used to cover the wound. In addition, of the two methods, the group with Duoderm CGF and an adhesive polyvinyl chloride film reduced the effect of wound contraction better than in the group with Duoderm CGF and Tegaderm. It is expected, therefore, that the skin fixation method that the authors presented could be applied to other researchers' wound healing experiments as a skin fixation method: for example, experiments on wound healing regulators, growth factors, and inflammatory mediators, and those comparing products that support the wound healing process.

The present study could also function as a reference in the design of future studies evaluating skin fixation methods by means of other materials.

\section{REFERENCES}

1. Broughton G 2nd, Janis JE, Attinger CE. The basic science of wound healing. Plast Reconstr Surg 2006;117:12S-34S.

2. Ehrlich HP, Needle AL. Wound healing in tight-skin mice: delayed closure of excised wounds. Plast Reconstr Surg 1983; 72:190-8.

3. Korula P, Varma SK, Sunderrao S. Inhibition of wound contraction by point-to-point adherent splintage. Plast Reconstr Surg 1995;95:725-30.

4. Callaghan MJ, Chang EI, Seiser N, et al. Pulsed electromagnetic fields accelerate normal and diabetic wound healing by increasing endogenous FGF-2 release. Plast Reconstr Surg 
2008;121:130-41.

5. Lin CD, Allori AC, Macklin JE, et al. Topical lineage-negative progenitor-cell therapy for diabetic wounds. Plast Reconstr Surg 2008; 122:1341-51.

6. Lindenblatt N, Calcagni M, Contaldo C, et al. A new model for studying the revascularization of skin grafts in vivo: the role of angiogenesis. Plast Reconstr Surg 2008;122:1669-80.

7. Berry DP, Harding KG, Stanton MR, et al. Human wound contraction: collagen organization, fibroblasts, and myofibroblasts. Plast Reconstr Surg 1998;102:124-31.

8. Welch MP, Odland GF, Clark RA. Temporal relationships of F-actin bundle formation, collagen and fibronectin matrix assembly, and fibronectin receptor expression to wound contraction. J Cell Biol 1990;110:133-45.
9. Aarabi S, Bhatt KA, Shi Y, et al. Mechanical load initiates hypertrophic scar formation through decreased cellular apoptosis. FASEB J 2007;21:3250-61.

10. Green MC, Sweet HO, Bunker LE. Tight-skin, a new mutation of the mouse causing excessive growth of connective tissue and skeleton. Am J Pathol 1976;82:493-512.

11. Engin C, Demirkan F, Ayhan S, et al. Delayed effect of denervation on wound contraction in rat skin. Plast Reconstr Surg 1996;98:1063-7.

12. Svensjo T, Pomahac B, Yao F, et al. Accelerated healing of full-thickness skin wounds in a wet environment. Plast Reconstr Surg 2000;106:602-12.

13. Vranckx JJ, Slama J, Preuss S, et al. Wet wound healing. Plast Reconstr Surg 2002;110:1680-7. 